\title{
Effects of small interfering RNA interference of connexin 37 on subcutaneous gastric tumours in mice
}

\author{
YUANMING JING ${ }^{1,2}$, SUXIA GUO $^{3}$, XIAOPING ZHANG ${ }^{1}$, AIJING SUN ${ }^{1}$, \\ FENG TAO $^{1}$, HAIXING JU ${ }^{4}$ and HAIXIN QIAN ${ }^{2}$
}

${ }^{1}$ Department of Gastrointestinal Surgery, Shaoxing People's Hospital, Shaoxing Hospital of Zhejiang University, Shaoxing, Zhejiang 312000; ${ }^{2}$ Department of General Surgery, The First Affiliated Hospital of Soochow University, Suzhou, Jiangsu 215006; ${ }^{3}$ Department of Cardiology, The Affiliated People's Hospital of Nanjing Medical University in Wuxi and People's Hospital of Wuxi City, Wuxi, Jiangsu 214023; ${ }^{4}$ Department of Colorectal Surgery, Zhejiang Provincial Tumor Hospital, Hangzhou, Zhejiang 310022, P.R. China

Received October 14, 2013; Accepted May 9, 2014

DOI: $10.3892 / \mathrm{mmr} .2014 .2609$

\begin{abstract}
The present study aimed to investigate the effects of small interfering (si)RNA interference of connexin 37 (Cx37) on subcutaneous gastric tumours in mice. Constructed lentiviruses carrying siRNA against $\mathrm{Cx} 37$ significantly knocked down Cx37 mRNA and protein expression in vitro. A total of 60 mice with gastric cancer were randomly divided into the Cx37 siRNA group, the mock-siRNA group and the control group. Cx37 siRNA, mock-siRNA and saline were separately injected (with the lentiviruses transfected into the gastric cancer cells). Following six weeks, the Cx 37 mRNA expression, Cx37 protein expression and tumor apoptosis were detected using semiquantitative reverse transcription-polymerase chain reaction, western blot analysis and terminal deoxynucleotidyl transferase-mediated dUTP nick end labelling, respectively. Six weeks following lentiviral transfection, the Cx37 mRNA levels in the Cx37 siRNA group, mock-siRNA group and saline group decreased to 42,63 and $67 \%$, respectively $(\mathrm{P}<0.05)$. The mock-siRNA group demonstrated no significant change in Cx37 levels compared with the control group. Western blot analysis revealed lower $\mathrm{Cx} 37$ protein levels in the $\mathrm{Cx} 37-\mathrm{RNAi}$ group than in the other groups $(0.21 \pm 0.07$ vs. $0.65 \pm 0.06$ vs. $0.54 \pm 0.07)$, and that the apoptotic index of the Cx37-RNAi group was higher than those of the mock-siRNA and control
\end{abstract}

Correspondence to: Professor Yuanming Jing, Department of Gastrointestinal Surgery, Shaoxing People's Hospital, Shaoxing Hospital of Zhejiang University, 568 Zhongxing Road, Shaoxing, Zhejiang 312000, P.R. China

E-mail: 13665103927@163.com; yuanmjcn@yeah.net

Dr Haixin Qian, Department of General Surgery, The First Affiliated Hospital of Soochow University, 296 Shizi Street, Suzhou, Jiangsu 215006, P.R. China

E-mail: haixinqian@126.com

Key words: gastric cancer, connexin 37, small interfering RNA groups $(19.7 \pm 5.1$ vs. $9.8 \pm 6.4$ vs. $10.5 \pm 7.2 \%, 11.1 \pm 6.9 ; \mathrm{P}<0.05)$. In conclusion, it was demonstrated that $\mathrm{Cx} 37$ siRNA is correlated with gastric cancer. Interference of Cx37 effectively reduces $\mathrm{Cx} 37$ mRNA and protein expression and promotes tumour apoptosis.

\section{Introduction}

Gastric cancer is the fourth most common type of cancer and the second leading cause of cancer-associated mortality worldwide (1-5), with nearly one million novel cases diagnosed annually. Gap junction proteins are present in cells responsible for information and substance exchange. Gap junction proteins are channel proteins essential for cell proliferation, differentiation and growth, and connexin (Cx) gene development (6-9). $\mathrm{Cx}$ has complex functions involving numerous physiological and pathological processes. This gene is correlated with tumour development and is thus termed 'the second type of tumour suppressor gene'. $\mathrm{Cx}$ is a multigene family and all members of the gene sequence have high degrees of homology. Currently, 13 different types of $\mathrm{Cx}$ genes have been identified in human tissues. A large number of studies (10-13) have demonstrated that $\mathrm{Cx}$ gene expression in various tumour cells is significantly reduced or is even completely inhibited, which results in the decrease or disappearance of the gap junctional intercellular communication (GJIC) function and eventually leads to malignant cell transformation and tumour formation. By contrast, other studies have demonstrated (14) that certain benign tumours, malignant tumours and transformed cells exhibit stable Cx gene expression. Conversely, other studies have revealed that certain $\mathrm{Cx}$ genes are not expressed in normal cells but are highly expressed in tumours (15). C1019T polymorphisms in the Cx37 gene lead to C-T mutations at the 1,019 position of the gene-coding region, resulting in missense mutations (proline-serine, $\mathrm{P}-\mathrm{S}$ ) during the encoding of 319 amino acids, which directly affects the formation of the three secondary structures of Cx37. Simultaneously, the original phosphorylation site of the $\mathrm{Cx} 37 \mathrm{C}$ end, particularly 
its functional area and its bond specificity to protein kinases and protein ligands, are altered due to the encoding of different amino acids. This change subsequently affects the activity of the gap junction protein $\mathrm{Cx} 37$. A study has demonstrated that Cx37 gene (C1019T) polymorphisms affect the function of GJIC (16) and may be involved in the growth of tumor cells. In our previous study (17), 592 patients with detected Cx37 C1019T gene polymorphisms were investigated. Of the total number of cases, 388 had gastric cancer and 204 had chronic superficial gastritis. The results demonstrated that in the gastric cancer group, $\mathrm{C}$ gene frequency was enhanced, whereas the $\mathrm{T}$ gene frequency significantly decreased, and that these changes were more pronounced in male patients. Following correcting for gender factors, the Cx37 C1019T $\mathrm{C}$ allele frequency in Helicobacter pylori (Hp)-positive gastric cancer patients increased, whereas the $\mathrm{T}$ gene frequency was significantly reduced. These results demonstrated a marked correlation between Cx37 C1019T gene polymorphisms and Hp-positive gastric carcinoma.

Previously, small interfering RNA (siRNA) has been effective in silencing target genes via RNA interference (RNAi) (18-21), a mechanism conserved in nature, from yeast to humans. Lentiviruses are able to efficiently deliver siRNA due to their ability to stably transduce dividing and non-dividing cells. In the present study, one lentiviral vector was constructed to knock down Cx37, with the aim of investigating its effects on gastric cancer in nude mice.

\section{Materials and methods}

Cell culture. The HEK293 cell line was cultured as previously described (22). The HEK 293 cells were derived from the cell bank of the Chinese Academy of Science and cultured in a complete medium (Gibco-BRL, Grand Island, NY, USA) at $37^{\circ} \mathrm{C}$ in a $5 \% \mathrm{CO}_{2}$ environment. When the cell fusion rate reached $90 \%$, the cells were allowed to proceed to the subsequent generation by 1:4 and were then maintained in the logarithmic growth phase. Recovery was performed when the cells demonstrated poor growth. The present study was conducted in strict accordance with the recommendations in the Guide for the Care and Use of Laboratory Animals of the National Institutes of Health. The animal use procedure was reviewed and approved by the Institutional Animal Care and Use Committee (IACUC) of the First Affiliated Hospital of Soochow University (Suzhou, China).

Lentiviral vector preparation and RNAi target screening. Three different sequences (sites A-C) of the Cx37 genes in mice were selected as targets for RNAi. The targeted sequences were as follows: mm-Cx37-si-1, 5'-GGUUAACGG UGCUCUUCAU-3', location 209; mm-Cx37-si-2,5'-CCAAGG ACCUACAUGUAGA-3', location 488; and mm-Cx37-si-3, 5'-CAGACCCUUACCCUGAACA-3', location 841. p3XFLAG-Cx37 and p3XFLAG were used as controls.

The cells were collected following $72 \mathrm{~h}$ and were then washed three times with cool phosphate-buffered saline (PBS). Residual PBS was removed by suctioning. Following this, $0.2 \mathrm{ml}$ radioimmunoprecipitation assay lysis buffer containing inhibitors of serine, cysteine and metalloproteinase was added. The resulting mixture was then placed on ice for $30 \mathrm{~min}$. The cells were collected using a cell scraper and placed in $1.5-\mathrm{ml}$ centrifuge tubes on ice. The cells were then ultrasonically lysed for $30 \mathrm{sec}$ at $4^{\circ} \mathrm{C}$. The supernate was centrifuged for $30 \mathrm{~min}$ at $22,559 \mathrm{x}$ g, transferred to another clean microcentrifuge tube and then stored at $-20^{\circ} \mathrm{C}$.

The protein concentrations were determined by the bicinchoninic acid (BCA) method using Pierce's BCA Protein Assay Reagent kit (Pierce Biotechnology, Inc. Rockford, IL, USA). Solutions A and B (50:1) were mixed and the resulting mixture was maintained at a room temperature for $30 \mathrm{~min}$. Following this, $200 \mu 1$ of the mixture was added to each hole of a 96-well plate, followed by $10 \mu \mathrm{l}$ of various concentrations of standard bovine serum albumin (dilution, 1:10). A blank sample containing $10 \mu \mathrm{l}$ of double-distilled water was also placed in a well, adjusted to zero and maintained at $37^{\circ} \mathrm{C}$ for $30 \mathrm{~min}$ in a microplate reader (BioTek Instruments, Inc., Winooski, VT, USA). The absorbance [optical density (OD) value] of this blank was then determined at $570 \mathrm{~nm}$. The standard value was subsequently used to prepare a standard curve to calculate the total protein concentration. The interference efficiency of siRNA was determined by western blot analysis. SDS-PAGE was performed using a concentration of $10 \%$. The volume of each sample was $30 \mu \mathrm{g}$. Anti-tag antibody protein flags were incubated overnight at $4^{\circ} \mathrm{C}$ and the GAPDH expression in the samples was monitored. The antibody ratios were as follows: flag antibodies: Rabbit pAb $=1: 2,000$ and GAPDH: Rabbit $\mathrm{pAb}=1: 10,000$.

The transfection reagent Lipofectamine $2000^{\mathrm{TM}}$ was purchased from Invitrogen Life Technologies (Carlsbad, CA, USA). A wild-type Lipofectamine 2000 plasmid Cx37 3 interference fragment was used. The negative control and p3XFLAG-Cx37 were co-transfected with the RNAi plasmid and then mixed at a molar ratio of 5:1.

The cells were divided into six groups: The experimental groups (overexpression of empty plasmid group, Cx37 plasmid, Cx37 overexpression of recombinant plasmid + different interference fragment); the transfection reagent group (Lipofectamine 2000); the plasmid group (p3XFLAG and p3XFLAG-Cx37-0.4 $\mu \mathrm{g}$ ) and the interference fragment group $(50 \mathrm{nM})$. The transfection time was $72 \mathrm{~h}$. The optimum interference fragment was selected on the basis of the western blotting results.

The lentiviral vectors expressing Cx37 were prepared using the BLOCK-It Lentiviral Pol II miR RNAi Expression system (Yingjun Biological Company, Shanghai, China) and green fluorescent protein (GFP; Catalog no. K4948-00; Invitrogen Life Technologies). A scrambled siRNA sequence (labelled mock-siRNA) with no known homology to mammalian genes served as a control.

Gastric cancer modelling in mice. SGC-7901 murine gastric cancer cell lines were purchased from the Agricultural Academy (Zhejiang, China). The cells were cultured in an RPMI-1640 complete medium. Sterilised test samples were added to a 96-well plates containing all of the tested cells (1x $10^{5}$ cells/well) to obtain a final concentration of P1 $(50,100$ and $200 \mu \mathrm{g} / \mathrm{ml}$ ). Dimethyl sulfoxide was used as a negative control. Following cultivation for 24,48 and $72 \mathrm{~h}$ at $37^{\circ} \mathrm{C}$ in a humidified $5 \% \mathrm{CO}_{2}$ incubator, the percentage of viable cells was determined by an MTT assay (Yingjun Biological 
Company). The reading absorbance was determined at $570 \mathrm{~nm}$ using a Benchmark microplate reader (Bio-Rad, Hercules, CA, USA). The inhibitory rate was calculated using the following formula: $\%$ inhibitory rate $=1$ - $($ mean absorbency in test wells)/(mean absorbency in control wells) x 100 .

Female BALB/c-nu/nu mice (age, six weeks) were purchased from Shanghai SLAC Laboratory Animal Co., Ltd. (Shanghai, China). All of the mice were housed under controlled conditions (12-h light/dark cycle, $60 \%$ humidity and $25 \pm 1^{\circ} \mathrm{C}$ ) with free access to a standard pellet diet and water. At day 0,32 mice were randomly divided into three groups and subcutaneously inoculated with $0.2 \mathrm{ml}$ of the murine gastric cancer cell line suspension $\left(2.5 \times 10^{7}\right.$ cells $\left./ \mathrm{ml}\right)$ at the forelimb, to establish the gastric carcinoma mouse model. Following tumour inoculation, the maximum (a) and minimum (b) diameters of the solid tumours were measured with a vernier calliper every three days. The tumor volumes were calculated using $V=a \times b^{2} / 2$. After six weeks, all of the mice were divided into three groups; the Cx37-RNAi, mock-siRNA and control groups. Each of the three groups were intratumorally injected with Cx37-RNAi, mock-siRNA and saline. At the end of the experiment, the tumours were excised from the mice, immediately weighed and then stored in $10 \%$ formalin. The samples were later sectioned using a microtome, dehydrated in graded alcohol, embedded in paraffin, stained with haematoxylin and eosin, and examined using a Lecia-DM2500 microscope (Leica Microsystems, Mannheim, Germany). Cx37 mRNA expression, Cx37 protein expression and tumor apoptosis in the tumor cells were detected using semiquantitative reverse transcription-polymerase chain reaction (23), western blot analysis and terminal deoxynucleotidyl transferase-mediated dUTP nick end labelling (24), respectively.

Statistical analysis. Data are expressed as the mean \pm standard deviation. Continuous variables between the two groups were compared using independent t-tests and $\chi^{2}$ tests. Multiple groups were compared using analysis of variance. All of the analyses were conducted using the SPSS 17.0 software for Windows (SPSS, Inc., Chicago, IL, USA). The proportions were compared using Fisher's exact test when the expected frequency was $<5$; otherwise, the $\chi^{2}$ test was used. $\mathrm{P}<0.05$ was considered to indicate a statistically significant difference.

\section{Results}

Gene silencing in vitro. The HEK293 cell line was transfected with lentivirus-based vectors expressing three different Cx37 siRNA. Gene silencing analysis demonstrated that the Cx37-site 2 and 3 lentivirus was the most effective vector in blocking Cx37 expression. Therefore, Cx37-site 3 lentiviruses were selected for the subsequent in vivo studies (Fig. 1).

Efficient transfection of lentiviruses in mouse gastric cancer. Previous local viral delivery to subcutaneous gastric cancer in mice resulted in an efficient transfection. GFP expression is used as an efficient and convenient monitoring tool for determining the transfection efficiency of the lentiviruses. In the present study, GFP fluorescence was detected in the gastric cancer samples one week following transfection to confirm siRNA transfection. Increased fluorescence was detected two

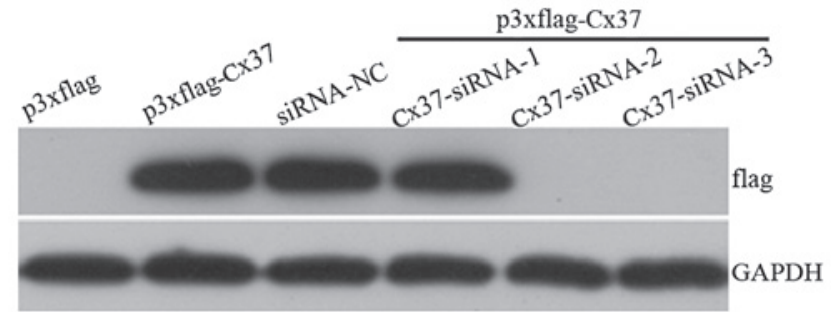

Figure 1. Western blot analysis of three different Cx37 siRNAs silencing in vitro. siRNA, small interfering RNA; Cx37, connexin 37.
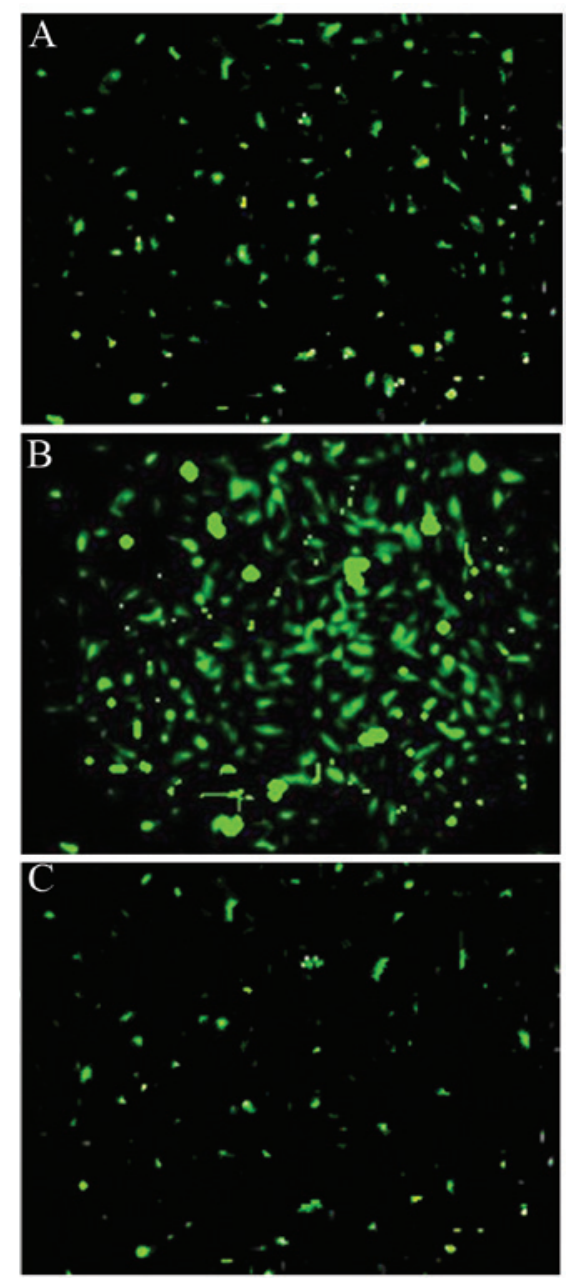

Figure 2. Lentiviral transfection efficiency observed by green fluorescent protein in gastric cancer cells at (A) 1 week; (B) 2 weeks; and (C) 6 weeks following transcfection.

weeks following transfection. A total of six weeks after the transfection, at the end of the study, a weak GFP remained visible (Fig. 2). These results demonstrate the efficient in vivo transfection of the lentiviruses by siRNA in gastric cancer. The local viral transfection did not affect the normal function of the animals and did not cause weight change (weight, 34.89 $\pm 4.16 \mathrm{~g}$ in transfected mice vs. $34.54+3.87$ in the mock-siRNA group and $31.96 \pm 4.84 \mathrm{~g}$ in the saline group).

Gene silencing in vivo. To examine the efficacy of the lentivirus-mediated gene silencing in vivo, the Cx37 mRNA and protein expression levels in the gastric cancer samples 


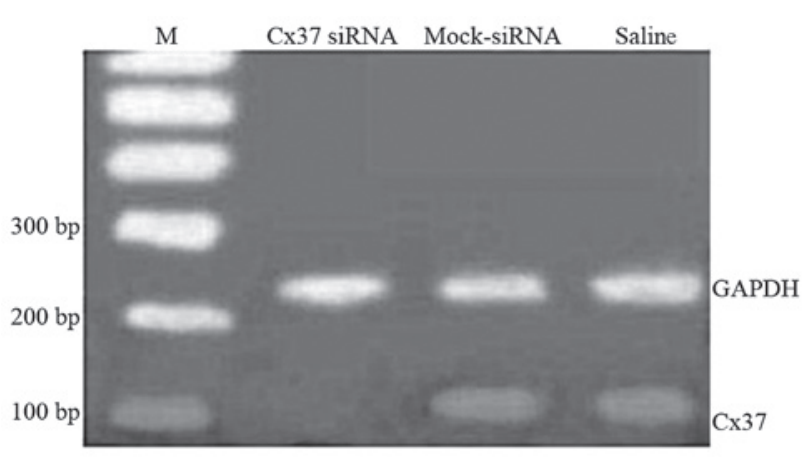

Figure 3. Electrophoresis results of $\mathrm{Cx} 37 \mathrm{mRNA}$ expression levels in gastric cancer cells in the three groups. siRNA, small interfering RNA; Cx37, connexin 37; bp, base pairs; M, marker.

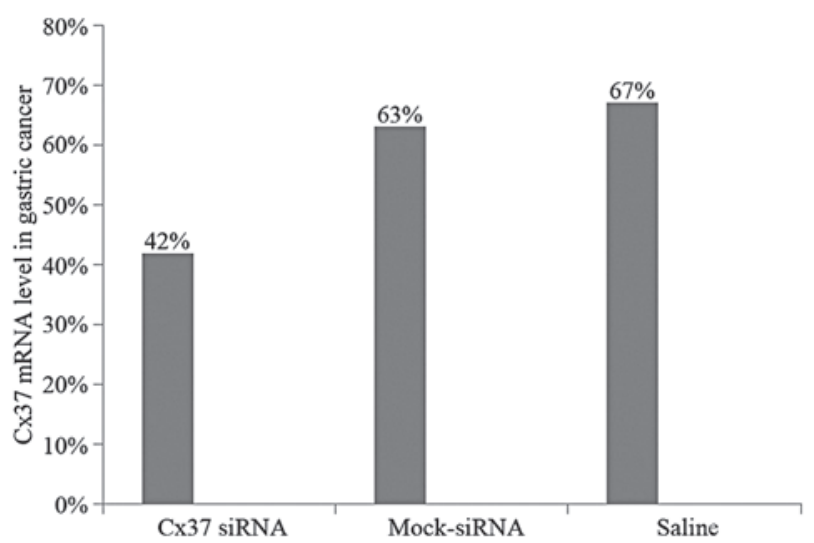

Figure 4. Cx37 mRNA expression analysis in gastric cancer cells in the three groups. siRNA, small interfering RNA; Cx37, connexin 37.

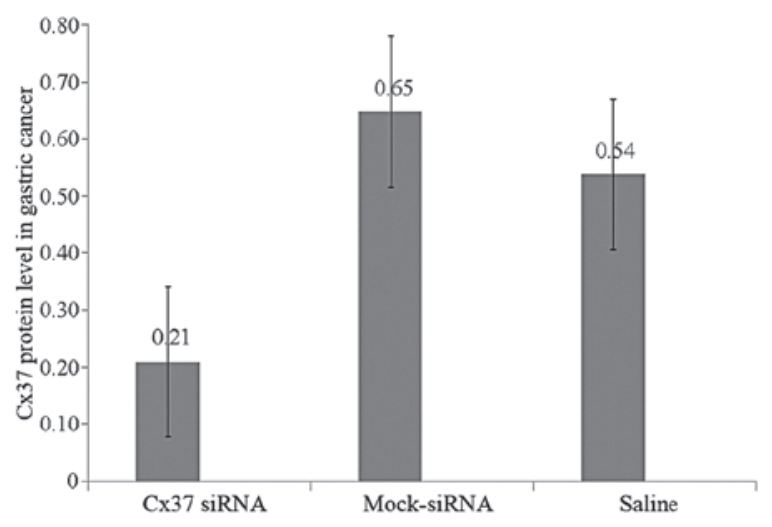

Figure 5. Cx37 protein level analysis in gastric cancer in the three groups. Data are expressed as the mean \pm standard deviation. siRNA, small interfering RNA; Cx37, connexin 37 .

were determined. The Cx37 mRNA levels of the Cx37 siRNA group, mock-siRNA group and saline group decreased to $42 \%$, by $63 \%, 67 \%$, respectively $(\mathrm{P}<0.05)$. The mock-siRNA group demonstrated no significant change in Cx37 compared with the control group. The western blot analysis results revealed that the $\mathrm{Cx} 37$ protein was lower in the $\mathrm{Cx} 37-\mathrm{RNAi}$ group than in the other groups $(0.21 \pm 0.07$ vs. $0.65 \pm 0.06$ vs. $0.54 \pm 0.07$; Figs. 3-5). Therefore, the local application of siRNA-lentiviruses efficiently silenced the target genes.

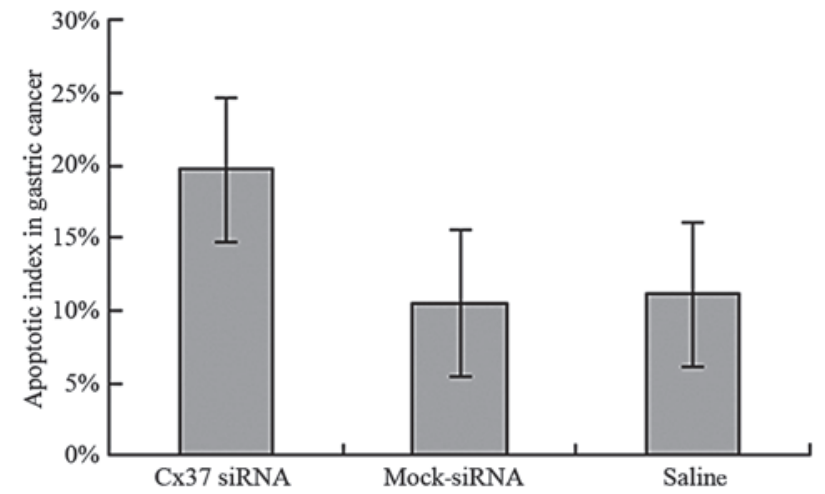

Figure 6. Apoptotic index in gastric cancer in the three groups. Data are expressed as the mean \pm standard deviation. siRNA, small interfering RNA; Cx37, connexin 37 .

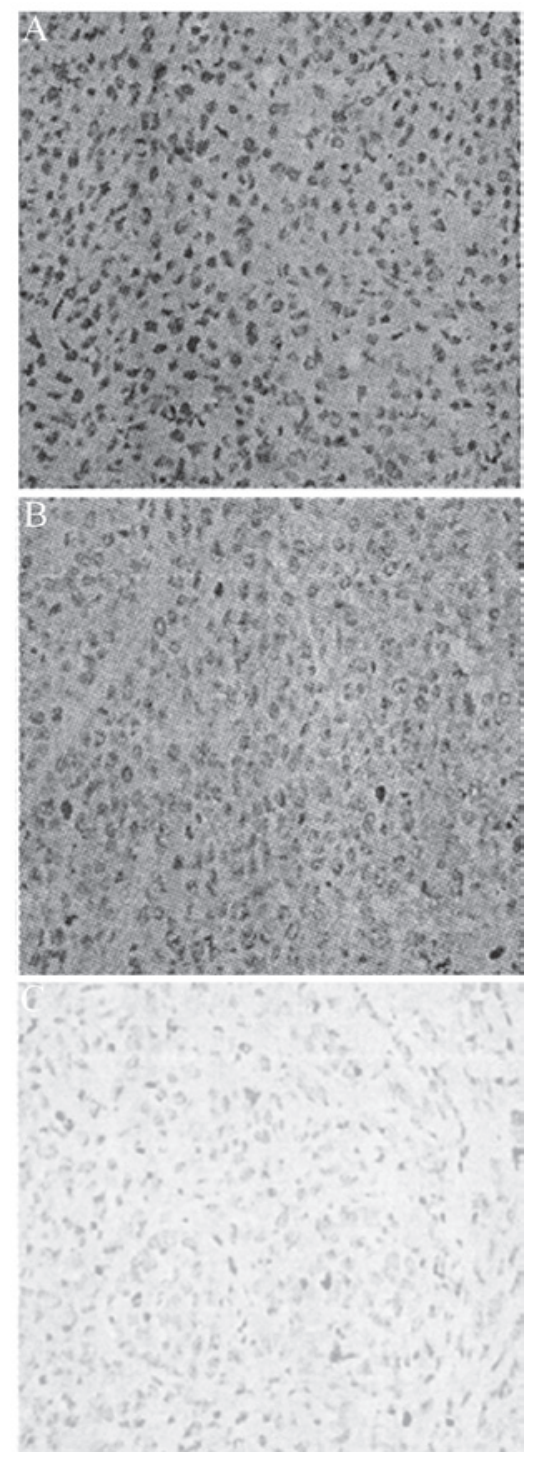

Figure 7. Apoptotic tumor cells in gastric cancer in the three groups (haematoxylin and eosin staining; magnification x10): (A) Cx37 siRNA;(B) mock siRNA; (C) saline. siRNA, small interfering RNA.

Gene knockdown effect on the apoptotic index in gastric cancer in mice. The apoptotic index of the Cx37-RNAi groups was higher than that of the mock-siRNA and control 
groups (19.7 \pm 5.1 vs. $9.8 \pm 6.4$ vs. $10.5 \pm 7.2 ; 11.1 \pm 6.9 \%$; $\mathrm{P}<0.05$; Figs. 6 and 7).

\section{Discussion}

In the development of genetic information in various organisms, homeobox genes encode and determine the location of information and are thus crucial in normal cellular control $(25,26)$. Gastric cancer is a multifactorial disease (3-5), and its pathogenesis, genetic and environmental factors are closely correlated. Our previous study (17) demonstrated that the Cx37 C1019T C allele frequency in Hp-positive gastric cancer patients increased, whereas the $\mathrm{T}$ allele frequency significantly decreased. Previously, epidemiological and experimental animal studies have demonstrated that chronic Hp infection is significantly correlated with gastric cancer $(27,28)$. A number of studies have also revealed that intermittently connected intercellular communication regulates the cell proliferation and differentiation mechanisms in gastric cancer $(29,30)$. In addition to passive diffusion, the intermittently connected intercellular communication may be adopted by small molecules including ions, small metabolites and secondary messenger molecules. $\mathrm{Cx}$ is a large family of gap-junction channel structure proteins. The murine Cx37 gene maps chromosome lp35.1 and encodes the 333 amino acids that form the Cx37 protein. In the present study, in contrast to the mouse $\mathrm{Cx} 37$ data in the gene bank, Mus musculus gap junction protein, $\alpha 4$, and mRNA (cDNA clone MGC:68218 IMAGE:4971608), completed the coding DNA sequence. Alignment statistics for Match \#1 Score Expect Identities Gaps Strand 1,866 bits (1010) 0.0 1012/1013 (99\%) 0/1013 (0\%) Plus/Plus demonstrated a highly homologous sequence. Three siRNAs were then constructed on the basis of the gene sequencing results. In addition, the western blot analysis results revealed that the silencing effects of the Cx37-site B and C lentiviruses were higher than that of the Cx37-site A virus. Therefore, the Cx37-site $\mathrm{C}$ was selected for the subsequent in vivo studies and the recombinant lentiviral vector was obtained. Then, the vector was injected into the gastric tumor and was successfully transfected. The results demonstrated that the Cx37 mRNA levels decreased by $42 \%$ compared with that of the mock and control groups, which were 63 and 58\%, respectively $(\mathrm{P}<0.05)$. The western blot analysis results revealed that $\mathrm{Cx} 37$ protein expression was lower in the Cx37-siRNA group than in the other groups $(0.21 \pm 0.07$ vs. $0.65 \pm 0.06$ vs. $0.54 \pm 0.07)$. Therefore, the local application of siRNA-lentiviruses efficiently silenced the target genes. At the same time, the apoptotic index of the Cx37-siRNA groups was higher than those of the mock-siRNA and control groups $(19.7 \pm 5.1$ vs. $9.8 \pm 6.4$ vs. $10.5 \pm 7.2 \%$; $\mathrm{P}<0.05)$. This result indicates that $\mathrm{Cx} 37-\mathrm{RNAi}$ promoted tumour cell apoptosis. However, further studies are required to elucidate the molecular mechanisms underlying this effect.

In conclusion, the present study demonstrated that lentivirus-mediated RNA interference effectively knocked down Cx37 genes in mice, which resulted in reduced Cx37 expression and an increased apoptosis index. Cx37 was demonstrated to be correlated with gastric cancer and this association may be used to develop novel therapeutic approaches for gastric cancer.

\section{References}

1. Crew KD and Neugut AI: Epidemiology of gastric cancer. World J Gastroenterol 12: 354-362, 2006.

2. Li GX and Hu YF: The current status and perspective of laparoscopic gastrectomy for gastric cancer. Zhonghua Wai Ke Za Zhi 51: 389-392, 2013 (In Chinese).

3. Tsai JS, Lin MT, Wu MS, et al: PBMC expressed adiponectin mRNA is predictive of survival in patients with gastric cancer. Clin Chem Lab Med 51: e313-316, 2013.

4. Chong ML, Loh M, Thakkar B, Pang B, Iacopetta B and Soong R: Phosphatidylinositol-3-kinase pathway aberrations in gastric and colorectal cancer: meta-analysis, co-occurrence and ethnic variation. Int J Cancer 134: 1232-1238, 2014.

5. Li HJ, Che XM, Zhao W, et al: Diet-induced obesity promotes murine gastric cancer growth through a nampt/sirt1/c-myc positive feedback loop. Oncol Rep 30: 2153-2160, 2013.

6. Völgyi B, Kovács-Oller T, Atlasz T, Wilhelm M and Gábriel R: Gap junctional coupling in the vertebrate retina: variations on one theme? Prog Retin Eye Res 34: 1-18, 2013.

7. Marandykina A, Palacios-Prado N, Rimkute L, Skeberdis VA and Bukauskas FF: Regulation of connexin36 gap junction channels by n-alkanols and arachidonic acid. J Physiol 591: 2087-2101, 2013.

8. Paul M, Wichter T, Gerss J, et al: Connexin expression patterns in arrhythmogenic right ventricular cardiomyopathy. Am J Cardiol 111: 1488-1495, 2013

9. Foley TT and Ehrlich HP: Through gap junction communications, co-cultured mast cells and fibroblasts generate fibroblast activities allied with hypertrophic scarring. Plast Reconstr Surg 131: 1036-1044, 2013.

10. Tencé M, Ezan P, Amigou E and Giaume C: Increased interaction of connexin 43 with zonula occludens-1 during inhibition of gap junctions by $\mathrm{G}$ protein-coupled receptor agonists. Cell Signal 24: 86-98, 2012.

11. Krutovskikh V, Mironov N and Yamasaki H: Human connexin 37 is polymorphic but not mutated in tumours. Carcinogenesis 17: 1761-1763, 1996.

12. Good ME, Nelson TK, Simon AM and Burt JM: A functional channel is necessary for growth suppression by Cx37. J Cell Sci 124: 2448-2456, 2011.

13. Burt JM, Nelson TK, Simon AM and Fang JS: Connexin 37 profoundly slows cell cycle progression in rat insulinoma cells. Am J Physiol Cell Physiol 295: C1103-C1112, 2008.

14. Simon KW, Roberts PC, Vespremi MJ, Manchen S and Schmelz EM: Regulation of beta-catenin and connexin-43 expression: targets for sphingolipids in colon cancer prevention. Mol Nutr Food Res 53: 332-340, 2009.

15. Cocciadiferro L, Miceli V, Kang KS, Polito LM, Trosko JE and Carruba G: Profiling cancer stem cells in androgen-responsive and refractory human prostate tumor cell lines. Ann NY Acad Sci 1155: 257-262, 2009.

16. Morel S, Burnier L, Roatti A, et al: Unexpected role for the human Cx37 C1019T polymorphism in tumour cell proliferation. Carcinogenesis 31: 1922-1931, 2010.

17. Jing YM, Guo SX, Zhang XP, Sun AJ, Tao F and Qian HX: Association between C1019T polymorphism in the connexin 37 gene and Helicobacter pylori infection in patients with gastric cancer. Asian Pac J Cancer Prev 13: 2363-2367, 2012.

18. Hou Q, Tang J, Wang Z, et al: Inhibitory effect of microRNA-34a on retinal pigment epithelial cell proliferation and migration. Invest Ophthalmol Vis Sci 54: 6481-6488, 2013.

19. Qin B, Chen Z, Jin W and Cheng K: Development of cholesteryl peptide micelles for siRNA delivery. J Control Release 172: 59-168, 2013

20. Li J and Mansmann UR: Modeling of non-steroidal anti-inflammatory drug effect within signaling pathways and miRNA-regulation pathways. PLoS One 8: e72477, 2013.

21. Yao Y, Charlesworth J, Nair V and Watson M: MicroRNA expression profiles in avian haemopoietic cells. Front Genet 4: $153,2013$.

22. Reinhard K, Rougier JS, Ogrodnik J and Abriel H: Electrophysiological properties of mouse and epitope-tagged human cardiac sodium channel Na v1.5 expressed in HEK 293 cells. F1000Res 2: 48, 2013.

23. Chen HJ, Zhu Z, Wang XL, et al: Expression of huCdc7 in colorectal cancer. World J Gastroenterol 19: 3130-3133, 2013

24. Zhu Q, Gu L, Wang Y, et al: The role of alpha-1 and alpha-2 adrenoceptors in restraint stress-induced liver injury in mice. PLoS One 9: e92125, 2014. 
25. Kuphal S, Wallner S and Bosserhoff AK: Impact of LIF (leukemia inhibitory factor) expression in malignant melanoma Exp Mol Pathol 95: 156-165, 2013.

26. Billingsley CN, Allen JR, Baumann DD, et al: Non-trisomic homeobox gene expression during craniofacial development in the Ts65Dn mouse model of Down syndrome. Am J Med Genet A 161: 1866-1874, 2013.

27. Ohnishi S, Ma N, Thanan R, et al: DNA damage in inflammation-related carcinogenesis and cancer stem cells. Oxid Med Cell Longev 2013: 387014, 2013
28. Kwak HW, Choi IJ, Cho SJ, et al: Characteristics of gastric cancer according to Helicobacter pylori infection status. J Gastroenterol Hepatol 29: 1671-1677, 2014.

29. Hosogi S, Kusuzaki K, Inui T, Wang X and Marunaka Y: Cytosolic chloride ion is a key factor in lysosomal acidification and function of autophagy in human gastric cancer cell. J Cell Mol Med 18: 1124-1133, 2014.

30. Oo HZ, Sentani K, Sakamoto N, et al: Overexpression of ZDHHC14 promotes migration and invasion of scirrhous type gastric cancer. Oncol Rep 32: 403-410, 2014 